\title{
Characterization of exosomes derived from Toxoplasma gondii and their functions in modulating immune responses
}

This article was published in the following Dove Press journal:

International Journal of Nanomedicine

\author{
Yawen $\mathrm{Li}^{1, *}$ \\ Yuan Liu',2,* \\ Fangming $\mathrm{Xiu}^{3}$ \\ Jianing Wang ${ }^{4}$ \\ Hua Cong' \\ Shenyi $\mathrm{He}^{1}$ \\ Yongyu $\mathrm{Shi}^{4}$ \\ Xiaoyan Wang ${ }^{4}$ \\ Xun $\mathrm{Li}^{5}$ \\ Huaiyu Zhou' \\ 'Department of Pathogen Biology, \\ School of Basic Medical Sciences, \\ Shandong University, Shandong, \\ People's Republic of China; ${ }^{2}$ Clinical \\ Laboratory, The People's Hospital \\ of Changle, Shandong, People's \\ Republic of China; ${ }^{3}$ Translational \\ Medicine, SickKids Research Institute, \\ the Hospital for Sick Children \\ Toronto, OT, Canada; ${ }^{4}$ Department \\ of Immunology, School of Basic \\ Medical Sciences, Shandong University, \\ Shandong, People's Republic of China; \\ ${ }^{5}$ Department of Medicinal Chemistry, \\ School of Pharmaceutical Sciences, \\ Shandong University, Shandong, \\ People's Republic of China \\ *These authors contributed equally \\ to this work
}

Introduction: Exosomes are nanograde membrane-bound vesicles secreted from most cell types through the fusion of multivesicular bodies with plasma membranes. Some of these exosomes are well defined, and are known to have immunomodulatory properties as well as play critical roles in intercellular communications. In this study, we characterized the exosomes derived from Toxoplasma gondii and their functions in aspect of immune responses.

Methods: T. gondii exosomes were isolated and identified using electron microscopy, nanoparticle tracking analysis, and Western blotting. The viability of macrophage RAW264.7 cells affected by exosomes was evaluated using a Cell Counting Kit (CCK-8). Then the uptake of $T$. gondii exosomes by RAW264.7 cells was detected by labeling with fluorescent dye PKH67. After exosomes stimulation, in vitro the production of interleukin (IL)-12, tumor necrosis factor (TNF)- $\alpha$, interferon (IFN)- $\gamma$ and IL-10 in RAW264.7 cells were investigated using enzyme-linked immunosorbent assay (ELISA). In immunized BALB/c mice, the antibodies, cytokines as well as the percentage of CD4+ and CD8+ T cells were determined using ELISA and flow cytometric analysis. Protective efficacy was evaluated by challenging intraperitoneally with tachyzoites of $T$. gondii. Results: We successfully isolated and characterized the exosomes derived from $T$. gondii. Functionally, the viability of macrophage RAW264.7 cells was significantly affected by exosomes at a high concentration $(160 \mu \mathrm{g} / \mathrm{mL})$. The production of IL-12, TNF- $\alpha$ and IFN- $\gamma$ in macrophage cells were increased, and the level of IL-10 was decreased. Furthermore, BALB/c mice immunized with $T$. gondii exosomes showed both humoral and cellular immune responses and also exhibited a prolonged survival time.

Conclusion: $T$. gondii exosomes could modulate macrophage activation in vitro and trigger humoral and cellular immune responses and partial protection against acute parasite infection in mice, which suggested that exosomes may serve as a potential candidate against toxoplasmosis. Keywords: Toxoplasma gondii, exosomes, macrophage, mouse, immune response

\section{Introduction}

Toxoplasma gondii is an obligate intracellular apicomplexan parasite which infects all warm-blooded mammals as well as birds and causes zoonotic toxoplasmosis., ${ }^{1,2}$ Approximately one-third of the world populations are infected with $T$. gondii and the majority of those infected are benign or asymptomatic. Even more serious, $T$. gondii infection can cause severe diseases in immunocompromised individuals with HIV/ AIDS or those who are being treated with chemotherapy. ${ }^{2,3}$ Furthermore, T. gondii infection causes congenital disease and abortions in human and domestic animals., Currently, treatment of toxoplasmosis depends primarily on chemotherapy. However, available drugs like sulfadiazine sodium have serious side effects, and toxoplasmosis
Correspondence: Huaiyu Zhou Department of Pathogen Biology, School of Basic Medical Sciences, Shandong University, Shandong, People's Republic of China Email zhouhy@sdu.edu.cn
International Journal of Nanomedicine 20 I8: 13 467-477

467

Dovepress f in 0

http://dx.doi.org/10.21 477/IJN.SI51/110 (c) (1) (2) $\odot 2018$ Li et al. This work is published and licensed by Dove Medical Press Limited. The full terms of this license are available at https:///www.dovepress.com/terms.php cc. hereby accept the Terms. Non-commercial uses of the work are permitted without any further permission from Dove Medical Press Limited, provided the work is properly attributed. For permission for commercial use of this work, please see paragraphs 4.2 and 5 of our Terms (https://www.dovepress.com/terms.php). 
may recur at any time. ${ }^{2}$ Although there is a commercial vaccine against $T$. gondii infection in sheep, which is effective in inducing the incidence of abortion, ${ }^{6}$ the vaccines against human toxoplasmosis are still unavailable.

Exosomes are tiny membrane-bound vesicles, approximately 30-100 nm in size, secreted from most cell types through the fusion of multivesicular bodies with plasma membrane. The vesicles contain a diverse suite of biomolecules including proteins, lipids, and nucleic acids, and some of them are known to have immunomodulatory properties. ${ }^{7,8}$ Moreover, as vehicles for delivery of cargos to recipient cells, exosomes are thought to play critical roles in intercellular communications. ${ }^{9,10}$ To date, numerous exosomes have been identified in both hematopoietic and non-hematopoietic cells, tumor cells, and microbes. Although the properties of exosomes vary depending on their biogenesis, their functions remain largely enigmatic.

In the past few years, several protozoan parasites including Leishmania species, Trypanosoma species, Plasmodium falciparum, and Trichomonas vaginalis have been found to release exosomes and/or microvesicles. ${ }^{8}$ Studies show that some exosomes secreted by these parasites have many physiological functions and are involved in many crucial biological activities. For instance, Leishmania exosomes could modulate the innate and adaptive immune responses in C57BL/6 mice by affecting monocytes and dendritic cells. ${ }^{11}$ In addition, these exosomes could deliver effector cargoes to the target cells during parasite early invasion via the host immunosuppression. ${ }^{12}$ Exosomes secreted by $T$. vaginalis have been found to play important roles in material exchanges and parasite adherence. ${ }^{13}$ A recent study by Wowk et al disclosed the first proteomic profiling of $T$. gondii extracellular vesicles (EVs) containing a broad range of canonical exosome proteins, however, the biological functions of exosomes from $T$. gondii are still largely unknown. ${ }^{14}$

Herein, we described the isolation and characterization of exosomes from $T$. gondii $\mathrm{RH}$ and ME49 strain in their biophysical and biological properties. Functionally, we found that $T$. gondii exosomes could modulate macrophage activation in vitro. Furthermore, $T$. gondii exosomes could trigger both humoral and cellular immune responses, and arouse partial protective immunity against acute $T$. gondii infection in BALB/c mice.

\section{Materials and methods Ethical statement}

All animal care procedures were conducted in strict accordance with the Animal Ethics Procedures and Guidelines of the People's Republic of China. The study was approved by the
Institutional Animal Care and Use Committee of Shandong University under Contract LL201602044, and all possible efforts were made to minimize distress on animals and the numbers of mice required for each experiment. Humane endpoints are considered to avoid the animals pain or suffering via euthanasia. Mice were monitored daily for signs of toxoplasmosis, such as fatigue, difficulty in feeding and severe ascites.

\section{Animal, parasite and cell}

Specific-pathogen-free (SPF) female BALB/c mice 6-8 weeks old were purchased from the Animal Centre of Shandong University, People's Republic of China. All the mice were allowed access to feeding and tap water ad libitum and were handled in strict accordance with good animal practice according to the animal ethics procedures.

The highly virulent tachyzoites of $T$. gondii RH strain were preserved in our laboratory at the School of Medicine, Shandong University, Shandong, People's Republic of China. The low virulent tachyzoites of $T$. gondii ME 49 strain were generously provided by the Department of Parasitology, Military Medical Sciences of the Military Veterinary Institute, Jilin, People's Republic of China. These two strains were used to prepare T. gondii exosomes and soluble tachyzoite antigens (STAg).

HEK293T cell line (Shanghai Institutes for Biological Sciences [SBIS], Shanghai, People's Republic of China) and RAW 264.7 macrophage cell line (SIBS) were maintained in complete medium supplemented with $10 \%$ fetal bovine serum (FBS), $100 \mathrm{IU} / \mathrm{mL}$ penicillin and $100 \mu \mathrm{g} / \mathrm{mL}$ streptomycin, at $37^{\circ} \mathrm{C}$, in a humidified chamber with $5 \% \mathrm{CO}_{2}$.

\section{Preparation of STAg}

Parasites were maintained by serial passage in confluent monolayers of human foreskin fibroblasts (HFF) under standard $T$. gondii culture conditions. As previously described, egressed parasites were harvested for the STAg preparation. ${ }^{15}$ In brief, the pelleted tachyzoites were resuspended in $0.1 \mathrm{M}$ phosphate buffered saline (PBS; pH 7.4) and freeze-thawed three times. After that, the lysate was sonicated on ice at $60 \mathrm{~W} / \mathrm{s}$ and centrifuged at $10,000 \times g$ for $30 \mathrm{~min}$. The supernatant containing STAg for protein quantification was determined via the Bradford method and stored at $-80^{\circ} \mathrm{C}$ until use.

\section{Isolation of exosomes}

Approximately, $100 \mathrm{~mL}$ of $T$. gondii culture media was performed for isolating exosomes using ExoEasy Maxi Kit (Qiagen, Hilden, Germany). Briefly, the collected tachyzoites of $T$. gondii were washed three times with sterile PBS, and centrifuged at $5,000 \times g$ for $10 \mathrm{~min}$, and resuspended in sterile 
PBS. Then, the parasites were cultured in Dulbecco's Modified Eagle's Medium (DMEM) without serum for $24 \mathrm{~h}$. After that, $100 \mathrm{~mL}$ of supernatants was filtered through $0.22 \mu \mathrm{m}$ membrane to remove the cell debris, potential membrane fragments from lysed cells and large vesicles (>500 nm). The filtrates were concentrated using a filter unit of Vivaflow 100 kDa MWCO PES (EMD Millipore, Billerica, MA, USA) to $10 \mathrm{~mL}$. Then, concentrated $T$. gondii culture media were precipitated for exosomes using ExoEasy Maxi Kit according to the manufacturer's instruction. To assess the concentration and size of exosomes, the exosome-sample was evaluated by nanoparticle tracking analysis (NTA) using the NanoSight NS300 (Malvern Instruments, Malvern, UK), and the sample was aliquoted $(50 \mu \mathrm{g} / \mathrm{vial})$ and stored at $-80^{\circ} \mathrm{C}$ until use.

\section{Electron microscopy}

Exosomes to be examined by transmission electron microscopy (TEM) were isolated and coated on to a formvar/carbon grid as previously described. ${ }^{16}$ Briefly, the exosomal samples were fixed with $2 \%$ glutaraldehyde and $2 \%$ paraformaldehyde in $0.1 \mathrm{~mol} / \mathrm{L}$ sodium cacodylate buffer at $\mathrm{pH} 7.3$ for $3 \mathrm{~h}$ at room temperature. Then, the grids were air dried and visualized using a JEM-1011 transmission electron microscope (JEOL, Tokyo, Japan) at $100 \mathrm{kV}$.

\section{Western blotting}

The exosomal samples $(20 \mu \mathrm{g})$ were boiled at $100^{\circ} \mathrm{C}$ for 5 min, subsequently separated in $10 \%$ sodium dodecyl sulfate polyacrylamide gel (SDS-PAGE) for $30 \mathrm{~min}$ and transferred to a polyvinylidene difluoride (PVDF) membrane for $110 \mathrm{~min}$. Then, the membrane was blocked with 5\% non-fat skim milk for $2 \mathrm{~h}$ at room temperature and probed with monoclonal antibody anti-P30 (Abcam, Cambridge, UK), anti-CD63 (Abcam) and anti-Hsp 70 (Abcam), respectively, at $4{ }^{\circ} \mathrm{C}$ overnight. Two different HRP-conjugated antibodies were used: Goat antimouse IgG antibody (1:10,000; Vazyme, Nanjing, People's Republic of China) and goat anti-Rabbit IgG antibody (1:7,500; EarthOx, San Francisco, CA, USA) as secondary antibody. Finally, after washing three times with phosphate buffered saline with Tween-20 (PBST), the membrane was soaked in DAB Reagents (Boshide Biotech Co, Wuhan, People's Republic of China) for signal development.

\section{Uptake of T. gondii exosomes by macrophages}

To examine whether $T$. gondii exosomes can be taken up by macrophages, the exosomes were labeled with the green fluorescent dye PKH67 (Sigma-Aldrich Co., St Louis, MO, USA) according to the manufacturer's directions with minor modifications. Briefly, the T. gondii exosomes were diluted and suspended in sterile PBS to a final concentration of $100 \mu \mathrm{g} / \mathrm{mL}$. PKH67 dye $(1.6 \mu \mathrm{L})$ was diluted in $500 \mu \mathrm{L}$ of Diluent C (PKH67 solution). Then, $500 \mu \mathrm{L}$ PKH67 solution and $200 \mu \mathrm{L}$ diluent exosomes were mixed in a $1.5 \mathrm{~mL}$ centrifugation tube. Samples were mixed gently for $5 \mathrm{~min}$ at $37^{\circ} \mathrm{C}$, and $800 \mu \mathrm{L} 1 \%$ bovine serum albumin (BSA) was added to bind the excess PKH67 dye. The PKH67-stained exosomes were washed three times in sterile PBS to remove excess dye and collected by ExoEasy Maxi Kit. Finally, PKH67-labeled exosomes were resuspended in DMEM. Macrophages were cultured on coverslips in 24-well plates at a mean of $3 \times 10^{5}$ cells/well. After $48 \mathrm{~h}$ incubation at $37^{\circ} \mathrm{C}$ in an atmosphere of $5 \% \mathrm{CO}_{2}$ with complete medium, the slides were washed three times in PBS to eliminate the influence of serum exosomes, and each medium containing $10 \mu \mathrm{L}$ of the PKH67-labeled exosomes or the same volume of the PKH67-PBS control was added into each well. Then, cells and $T$. gondii exosomes were cultured for $2 \mathrm{~h}$, and the coverslips were gently washed with PBS and fixed with 4\% paraformaldehyde solution for $15 \mathrm{~min}$ at room temperature and washed three times with PBS. For the fluorescence microscopy, nucleus staining was performed using a ProLong Gold Antifade Reagent with DAPI (4',6-diamidino2-phenylindole; Solarbio, Beijing, People's Republic of China). Finally, the coverslips were removed from the wells and placed on slides for viewing on a fluorescence microscope (Carl Zeiss Meditec AG, Jena, Germany), operated by Axiovision software (Carl Zeiss Meditec AG).

\section{Macrophage viability assay}

The cell viability in vitro was evaluated using a Cell Counting Kit (CCK-8) purchased from Dojindo Laboratories, Kumamoto, Japan according to manufacturer's directions. Briefly, exosomes were diluted to a concentration of $10 \mu \mathrm{g} / \mathrm{mL}$, $20 \mu \mathrm{g} / \mathrm{mL}, 40 \mu \mathrm{g} / \mathrm{mL}, 80 \mu \mathrm{g} / \mathrm{mL}$ and $160 \mu \mathrm{g} / \mathrm{mL}$, respectively, in sterile PBS. The macrophage RAW264.7 cells were plated in 48 -well plates $\left(5 \times 10^{6}\right.$ cells/well), and incubated at $37^{\circ} \mathrm{C}$ for $24 \mathrm{~h}$ under $5 \% \mathrm{CO}_{2}$. The cells were stimulated with $100 \mu \mathrm{L}$ exosomes at different concentrations or PBS alone, and incubated under the same conditions for an additional $24 \mathrm{~h}$. After incubation, $10 \mu \mathrm{L}$ of CCK-8 reagent was added to each well and incubation continued for another $4 \mathrm{~h}$. Finally, the absorbance was measured at $450 \mathrm{~nm}$ using an automatic enzyme analyzer (Allsheng, Hangzhou, People's Republic of China).

\section{Macrophage cytokine assay}

The macrophages were obtained as described above and cultured in triplicate in 48 -well plates $\left(5 \times 10^{6}\right.$ cells/well). 
Then, macrophages were incubated with $1 \times 10^{5}$ parasites, $10 \mu \mathrm{g} T$. gondii exosomes, $10 \mu \mathrm{g}$ BSA, and $50 \mu \mathrm{L}$ sterile PBS, respectively. After $24 \mathrm{~h}$ incubation, the cell-free supernatants were collected and analyzed for interleukin-10 (IL-10), IL-12, interferon- $\gamma$ (IFN- $\gamma$ ) and tumor necrosis factor- $\alpha$ (TNF- $\alpha)$. The assays were determined by specific ELISA assay kits (Bio-Swamp, Wuhan, People's Republic of China) according to the manufacturer's directions.

\section{Immunization and challenge}

To assess the immunogenicity of $T$. gondii exosomes, BALB/c mice were randomly assigned into four groups (14 per group). Before inoculation, $T$. gondii exosomes were diluted and suspended in sterile PBS to a final concentration of $100 \mu \mathrm{g} / \mathrm{mL}$. Three groups of mice were injected intramuscularly with $100 \mu \mathrm{L} \mathrm{T}$. gondii exosomes from RH strain (Exo-R), ME 49 strain (Exo-M) or PBS, respectively. Another group of 14 mice were set as blank controls without injection. All groups of mice were immunized three times on days 0,14 and 28 using the same dosages. Blood was collected through retro-orbital plexus puncture before each immunization and 2 weeks after the final immunization (on day 42), and stored at $-20^{\circ} \mathrm{C}$ until used for serological tests.

Two weeks after the last immunization (on day 42), four mice per group were sacrificed and their splenocytes were aseptically harvested for cytokine detection and flow cytometric analysis. The other ten mice in each group were challenged with $1 \times 10^{3}$ tachyzoites of $T$. gondii $\mathrm{RH}$ strain (PBS or Exo-R group) or ME-49 strain (Blank control or Exo-M group).

\section{Antibody assay}

The levels of antibody against $T$. gondii (total IgG, IgG1, IgG2a) in mouse sera were determined using ELISA assay as previously described. ${ }^{16}$ Briefly, STAg was diluted to $12.5 \mu \mathrm{g} / \mathrm{mL}$ in $50 \mathrm{mM}$ carbonate buffer ( $\mathrm{pH} 9.6)$ and divided into a 96-well enzyme panel with $50 \mu \mathrm{L}$ per well at $4^{\circ} \mathrm{C}$ overnight. Then, the plates were washed three times with PBST. The serum samples were diluted in $1 \% \operatorname{PBST}(1: 100)$ and $50 \mu \mathrm{L}$ placed into each well. Plates were incubated at $37^{\circ} \mathrm{C}$ for $60 \mathrm{~min}$ with gentle shaking. After washing three times, the wells were incubated with $100 \mu \mathrm{L}$ diluted enzyme-labeled second antibody (IgG-HRP, IgG1-HRP or IgG2a-HRP, 1:1,000 dilution) (Proteintech, Chicago, IL, USA) for $60 \mathrm{~min}$ at $37^{\circ} \mathrm{C}$. The reaction was stopped by adding $50 \mu \mathrm{L}$ stop solution 15 min later and the absorbance was measured at $450 \mathrm{~nm}$ using an automated ELISA reader (Allsheng).

\section{Cytokine assays from splenocytes}

To assay cytokine production levels, splenocytes were prepared and determined by ELISA as previously described. ${ }^{15}$ Briefly, the culture supernatants of splenocytes from each group 2 weeks after the final immunization were harvested and tested for IL-4 at 24 h, IL-10 and IL-12 at 72 h, and IFN- $\gamma$ at 96 h, using specific ELISA assay kits (Bio-Swamp) according to the manufacturer's directions.

\section{Flow cytometry assay}

To evaluate the subsets of $\mathrm{CD}^{+}$and $\mathrm{CD} 8^{+} \mathrm{T}$ cells in the spleens of immunized mice, flow cytometry assay was performed as previously described. ${ }^{15}$ Briefly, $10^{6}$ splenocytes were suspended in $100 \mu \mathrm{L}$ PBS prior to incubation with anti-mouse-CD3e-PE-cy5.5 (eBioscience, San Diego, CA, USA), anti-mouse-CD4-FITC (eBioscience) and antimouse-CD8-PE (eBioscience) at $4^{\circ} \mathrm{C}$ for $30 \mathrm{~min}$ without light. After washing with $2 \mathrm{~mL}$ PBS, cells were resuspended in PBS and analyzed with a FL500 flow cytometer (Beckman Coulter Inc., Brea, CA, USA). List mode data files were analyzed using FlowJo7.6 software. The total lymphocytes gate was defined by forward and side scatter in mouse splenocytes. Then, the $\mathrm{CD}^{+} \mathrm{T}$ lymphocytes gate was defined by side scatter and anti-mouse-CD3e-PE-cy5.5. The ratio of $\mathrm{CD}^{+}{ }^{+} \mathrm{CD} 4^{+} \mathrm{T}$ cells $/ \mathrm{CD}^{+}{ }^{+} \mathrm{CD} 8^{+} \mathrm{T}$ cells was calculated in at least three independent experiments that provided similar results.

\section{Statistical analysis}

All statistical analyses in this study, including proliferation assay, antibody response, cytokine assay, and flow cytometry assay were compared between groups by using one-way analysis of variance. Survival time for the mice was compared using the Kaplan-Meier method. All the analyses were carried out using SPSS 11.5 software at 95\% confidence level. For all analyses, $p<0.05$ was considered significant. Every experiment was done in triplicate and three independent experiments on different days were done as biological replicates.

\section{Results}

\section{Isolation and characterization of exosomes from $T$. gondii}

To characterize the exosomes secreted by $T$. gondii, about $80 \mu \mathrm{g}$ of vesicles were obtained from $8 \times 10^{6} \mathrm{~T}$. gondii tachyzoites in $100 \mathrm{~mL}$ of culture media. TEM analysis showed that these vesicles had intact continuous bilayer membranes, about 10-150 nm in diameter (Figure 1A). Furthermore, the size distribution of vesicles was determined 
A

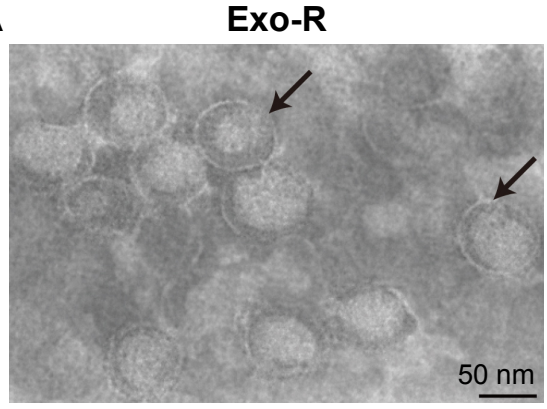

Exo-M

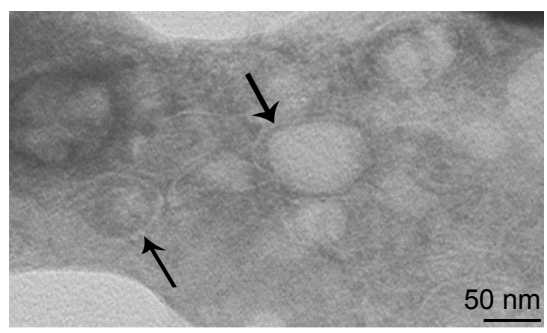

B

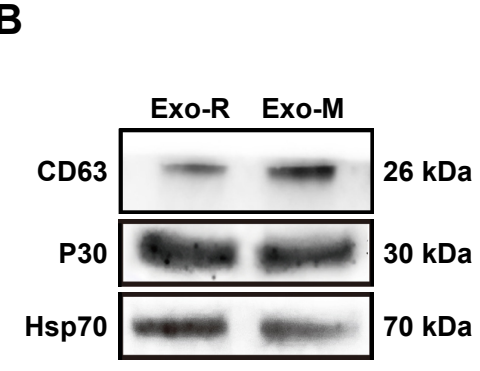

D

\begin{tabular}{lll}
\hline $\begin{array}{l}\text { Particle } \\
\text { size }(\mathbf{n m})\end{array}$ & $\begin{array}{l}\text { Population } \\
\text { (Exo-R) \% }\end{array}$ & $\begin{array}{l}\text { Population } \\
\text { (Exo-M) \% }\end{array}$ \\
\hline $1-50$ & 81.3 & 79.3 \\
$51-100$ & 12.7 & 17.2 \\
$101-150$ & 5.6 & 3.2 \\
$151-500$ & 0 & 0 \\
\hline
\end{tabular}

Figure I Characterization of Toxoplasma gondii-derived exosomes.

Notes: (A) Representative TEM images of vesicles derived from T. gondii RH strain or ME49 strain showing a range of exosomal morphologies (original magnification, $\times$ I00,000; scale bar $=50 \mathrm{~nm}$ ). Bilayer membranes are arrowed. TEM was performed at least three times. (B) Western blotting analysis of $T$. gondii-derived vesicles with anti-CD63, anti-P30 (anti-SAGI) and Hsp70. (C) Size distribution of purified vesicles derived from T. gondii using nanoparticle tracking analysis and showing a mean diameter of 50 nm. (D) Table from NanoSight NS300 analysis of percentage of purified vesicles derived from $T$. gondii in various size ranges.

Abbreviations: Exo-R, exosomes from RH strain; Exo-M, exosomes from ME 49 strain; TEM, transmission electron microscopy.

using nanoparticle tracking analysis. As shown in Figure 1C and $\mathrm{D}$, the size of the vesicles peaked at a mean diameter of $50 \mathrm{~nm}$, which is the typical morphological characteristic of exosomes. Western blotting results showed that the vesicles were positive for CD63, Hsp70 and P30 staining, which are specific markers for the exosomes (Figure 1B).

\section{Uptake of T. gondii exosomes by macrophages}

To determine whether $T$. gondii exosomes can be taken up by host cells, $T$. gondii exosomes were labeled with PKH67 dye (green fluorescence) and incubated with macrophage RAW264.7 cells in vitro. As shown in Figure 2, in the experimental group, the green fluorescence was observed in the cytoplasm of cells (Figure 2A), indicating that a large number of exosomes were taken up by the macrophages, compared with the PKH67-PBS control, or the PBS control (Figure 2B and C).

\section{Effect of $T$. gondii exosomes on the cell viability of macrophage RAW264.7 cells in vitro}

To determine the appropriate concentration of the exosomes used in the in vitro experiments, we investigated the influence of $T$. gondi exosomes on the viability of RAW264.7 cells using CCK-8 assay. As shown in Figure 3, the T. gondii exosomes did not affect the cell viability at concentrations lower than $80 \mu \mathrm{g} / \mathrm{mL}$. However, the cell viability was significantly reduced at the concentration of $160 \mu \mathrm{g} / \mathrm{mL}(p<0.05$ vs untreated cells). Thus, the appropriate concentration of $T$. gondii exosomes in vitro for RAW264.7 cells was below $80 \mu \mathrm{g} / \mathrm{mL}$.

\section{T. gondii exosomes affecting macrophage cytokine production in vitro}

To examine the cytokine variations during $T$. gondii exosomes stimulation, supernatants of the RAW264.7 cells were collected. As shown in Figure 4, the production of IL-12, TNF- $\alpha$ and IFN- $\gamma$ was significantly increased after T. gondii exosomes stimulation ( $p<0.05$ vs PBS or BSA control), which was comparable to that in the parasite infection group (Figure 4B-D). Meanwhile, the level of IL-10 was significantly down-regulated ( $p<0.05$ vs PBS or BSA control), which is shown in Figure 4A.

\section{Serum antibody responses in BALB/c mice to $T$. gondii exosomes}

As shown in Figure 5, significantly high levels of total IgG against $T$. gondii were detected in the sera from mice immunized with Exo-M or Exo-R, 4 and 6 weeks after the first inoculation, compared with that of the controls ( $p<0.05$ or $p<0.01)$. However, there were no significant differences in 
A

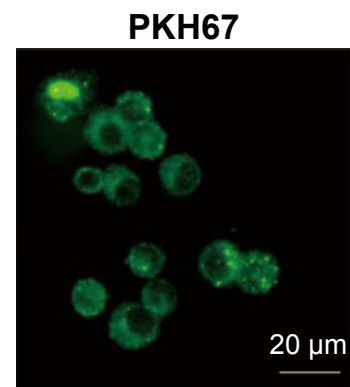

B

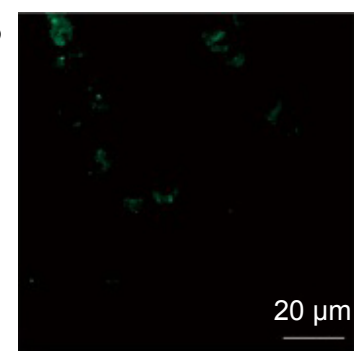

C

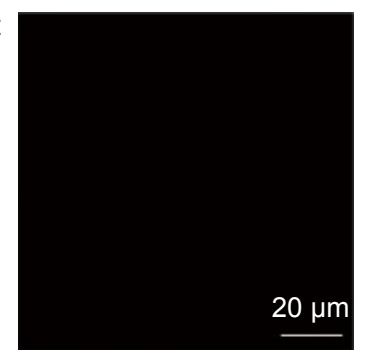

DAPI
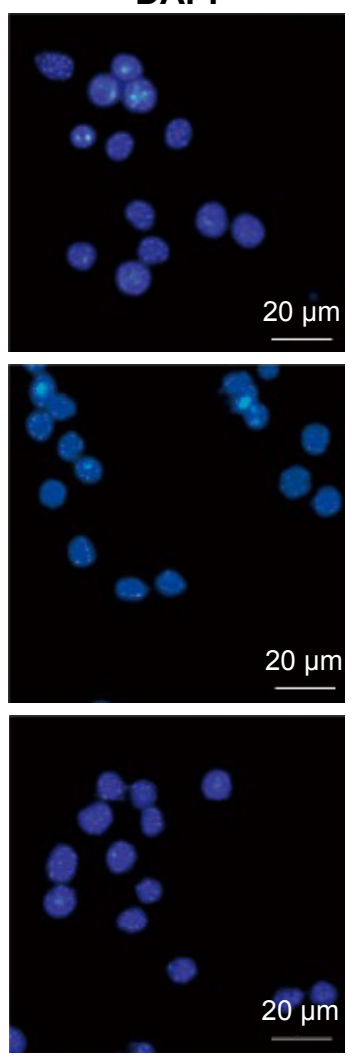

Merge
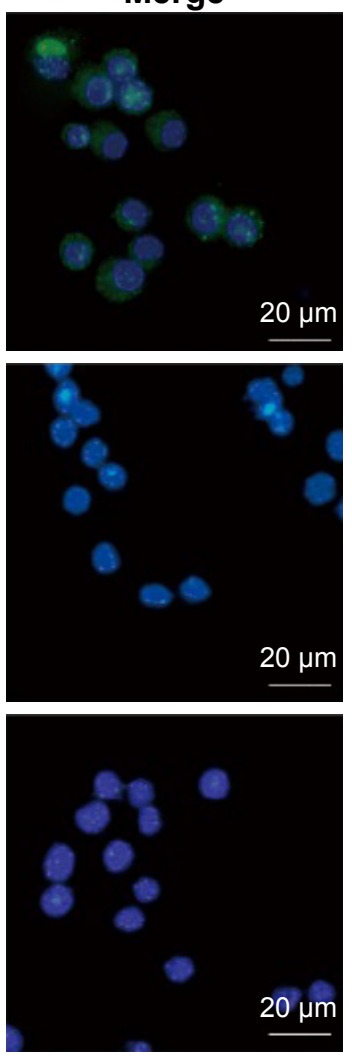

Figure 2 Uptake of Toxoplasma gondii exosomes by macrophages.

Notes: Ten micrograms of the (A) PKH67-labelled T. gondii exosomes, or (B) PKH67-PBS control, or (C) PBS control alone were added to the macrophages and incubated at $37^{\circ} \mathrm{C}$ for $2 \mathrm{~h}$. In the fluorescence microscopy pictures, PKH67 was used to label the exosomes (green) and DAPI was used to detect the nucleus of the macrophages (blue). Results are representative of three independent experiments.

Abbreviations: PBS, phosphate buffered saline; DAPI, 4',6-diamidino-2-phenylindole.

the total IgG levels between Exo-M and Exo-R groups, as well as that between the PBS and Blank controls (Figure 5A).

The levels of serum IgG1 and IgG2a against $T$. gondii were also detected 2 weeks after the last inoculation. The results



Figure 3 Effect of Toxoplasma gondii exosomes on the cell viability of macrophage RAW264.7 cells in vitro.

Notes: RAW264.7 cells were cultured with Exo-R or Exo-M $(0-160 \mu \mathrm{g} / \mathrm{mL})$ for $24 \mathrm{~h}$. Cell viability was determined by the CCK-8 assay. ${ }^{*} p<0.05$ compared with $0 \mu \mathrm{M}$ exosomes. One of three experiments with similar results is shown.

Abbreviations: Exo-R, exosomes from RH strain; Exo-M, exosomes from ME 49 strain; CCK-8, Cell Counting Kit. showed that an obvious predominance of IgG2a over IgG1 was observed in the immunized mice $(p<0.05)$ (Figure 5B and C), suggesting that a Th1-type cell immune response was elicited by immunization with $T$. gondii exosomes.

\section{Cellular immune responses in BALB/c mice to T. gondii exosomes}

The cytokines produced by splenocytes from immunized mice were evaluated by ELISA. As shown in Table 1, there were significantly high levels of both IFN- $\gamma$ and IL-12 $(p<0.05)$ in the Exo-M and Exo-R groups compared with those in the PBS or Blank control groups. However, there were no significant differences in the levels of IL-4 and IL-10 among all the groups.

The percentages of $\mathrm{T}$ cell subsets $\mathrm{CD} 4^{+}$and $\mathrm{CD} 8^{+}$in the spleen of immunized mice were evaluated using flow cytometry techniques. As shown in Figure 6, the percentage of $\mathrm{CD} 8^{+} \mathrm{T}$ cells in the Exo-M or Exo-R groups was higher than that in the PBS or Blank control groups $(p<0.05)$. However, there was no significant difference in the percentage of $\mathrm{CD} 4^{+}$ $\mathrm{T}$ cells between the Exo-M and Exo-R groups. 
A

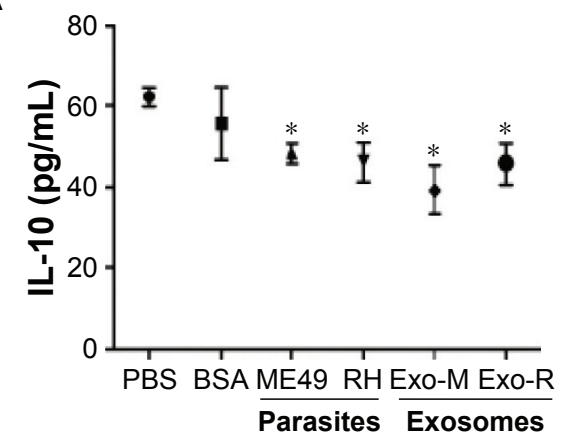

C

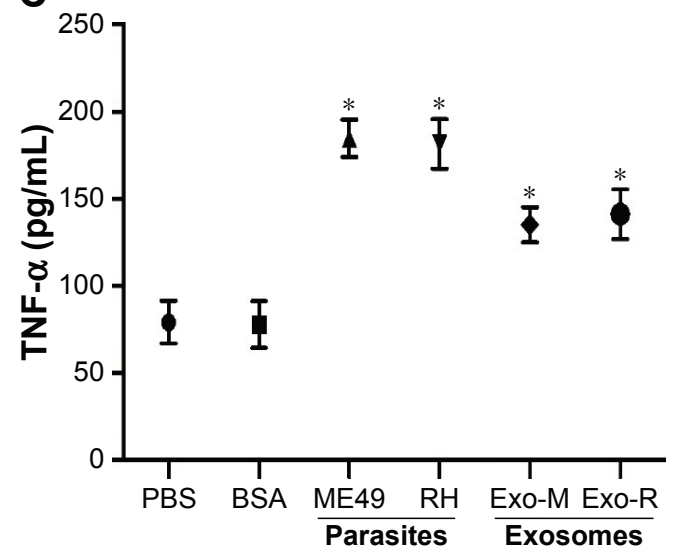

B

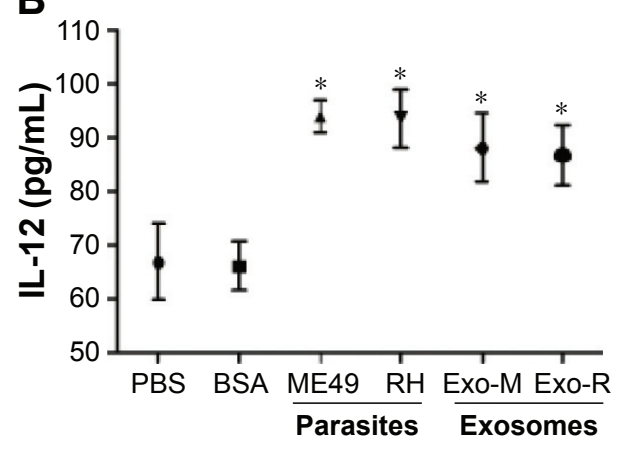

D

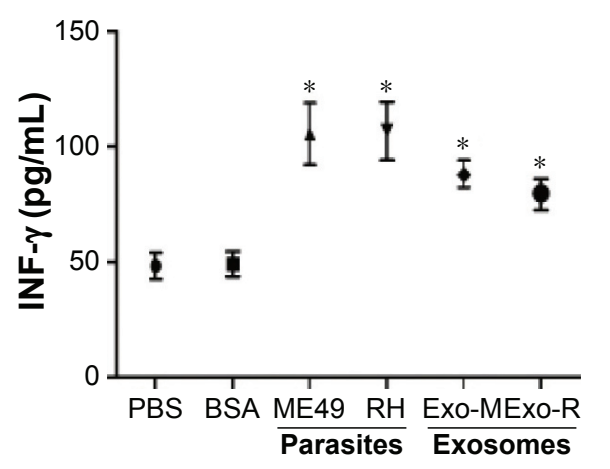

Figure 4 Macrophage cytokine productions in vitro by Toxoplasma gondii exosomes.

Notes: The supernatants of RAW264.7 cells were collected and assayed by ELISA for (A) IL-10, (B) IL-12, (C) TNF- $\alpha$, and (D) IFN- $\gamma$ at 24 h. *p $<0.05$ compared with PBS or BSA. One of three experiments with similar results is shown.

Abbreviations: Exo-R, exosomes from RH strain; Exo-M, exosomes from ME 49 strain; IL, interleukin; TNF, tumor necrosis factor; IFN, interferon; PBS, phosphate buffered saline; BSA, bovine serum albumin; ELISA, enzyme-linked immunosorbent assay.

\section{Protective immunity of $T$. gondii exosomes in mice}

The survival curves of mice after lethal challenge infections are shown in Figure 7. Non-immunized control mice were all dead within 8 days after T. gondii infection. However, the mice immunized with Exo-M were dead within 23 days post ME49 strain challenge, which showed a significantly prolonged survival time $(p<0.05)$. The mice immunized with Exo-R were all dead within 17 days post $\mathrm{RH}$ strain challenge, which also showed a significantly prolonged survival time compared with the control group $(p<0.05)$. But the difference in survival time of immunized mice between the Exo-M and Exo-R groups was not statistically different.



B

C
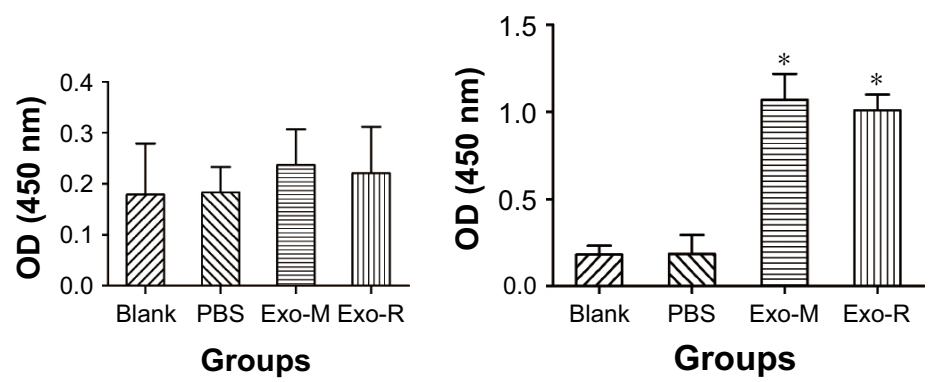

Figure 5 Antibody responses in immunized BALB/c mice.

Notes: (A) Total lgG antibody against Toxoplasma gondii in the sera of BALB/c mice immunized with T. gondii Exo-R or Exo-M, PBS and Blank control on weeks 0, 2, 4, 6 after the first inoculation. (B) IgGI, and (C) IgG2a against $T$. gondii in the sera of the immunized BALB/c mice 2 weeks after the last inoculation. Results are expressed as the mean OD $450 \pm$ SD and are representative of three individual experiments. $n=14$ animals per group, $* p<0.05$ or $* * p<0.01$ compared with PBS or Blank control. Abbreviations: Exo-R, exosomes from RH strain; Exo-M, exosomes from ME 49 strain; PBS, phosphate buffered saline. 
Table I Cytokine production by splenocytes of immunized $B A L B / c$ mice ( $n=4$ animals per group)

\begin{tabular}{lllll}
\hline Groups & \multicolumn{4}{l}{ Production of cytokines $(\mathrm{pg} / \mathrm{mL})$} \\
\cline { 2 - 5 } & IFN- $\boldsymbol{\gamma}$ & IL-4 & IL-10 & IL- I 2 \\
\hline Blank & $160.4 \pm 7.38$ & $93.9 \pm 2.04$ & $74.0 \pm 9.62$ & $97.1 \pm 3.8$ \\
PBS & $163.0 \pm 5.27$ & $91.1 \pm 3.08$ & $76.2 \pm 5.70$ & $99.5 \pm 5.4$ \\
Exo-M & $903.4 \pm 13.27 * *$ & $96.25 \pm 6.69$ & $78.4 \pm 2.79$ & $199.3 \pm 8.9 *$ \\
Exo-R & $879.0 \pm 6.8 I^{* *}$ & $92.43 \pm 3.78$ & $81.9 \pm 4.50$ & $204.6 \pm 3.2^{*}$
\end{tabular}

Notes: Splenocytes from mice were harvested 2 weeks after the last immunization and assayed for IL-4 at 24 h, IL- 10 and IL- 12 at 72 h, and IFN- $\gamma$ at 96 h. Results are expressed as mean \pm SD from three independent experiments. ${ }^{*} p<0.05$ or $* * p<0.0$ l compared with PBS or Blank control.

Abbreviations: Exo-R, exosomes from RH strain; Exo-M, exosomes from ME 49 strain; IL, interleukin; IFN, interferon; PBS, phosphate buffered saline.

\section{Discussion}

Previous studies have looked at properties and functions of exosomes associated with $T$. gondii, however, those exosomes were from $T$. gondii-infected cells, such as dendritic cells (DCs), macrophages, HFF or rat myoblast L6 cells. ${ }^{17-21}$
A recent study has demonstrated that $T$. gondii EVs contain some canonical exosome proteins, however, their biological functions have not been investigated. ${ }^{14}$ In the present study, we isolated and characterized $T$. gondii-derived EVs using TEM, NTA and Western blotting analysis. Consistent with the general observation, the vesicles isolated from $T$. gondii have an intact continuous bilayer membrane, ranging from $10-150 \mathrm{~nm}$ with a mean size of $50 \mathrm{~nm}$ in diameter, which are not apoptotic bodies. Generally, exosomes are characterized based on their protein content, which indicates their endosomal origin. ${ }^{9,10}$ Our results showed that the vesicles were positive for CD63, Hsp70, and P30 using Western blotting. Conventionally, CD63 and Hsp70 are known as exosome markers in many cell types ${ }^{9,10}$ as well as in $T$. gondii, while protein P30, namely a major surface antigen 1 (SAG1) of $T$. gondii, is an extremely useful marker for identifying T. gondii. ${ }^{22}$ Collectively, our results confirmed that the microvesicles excreted from $T$. gondii were exosomes in
A
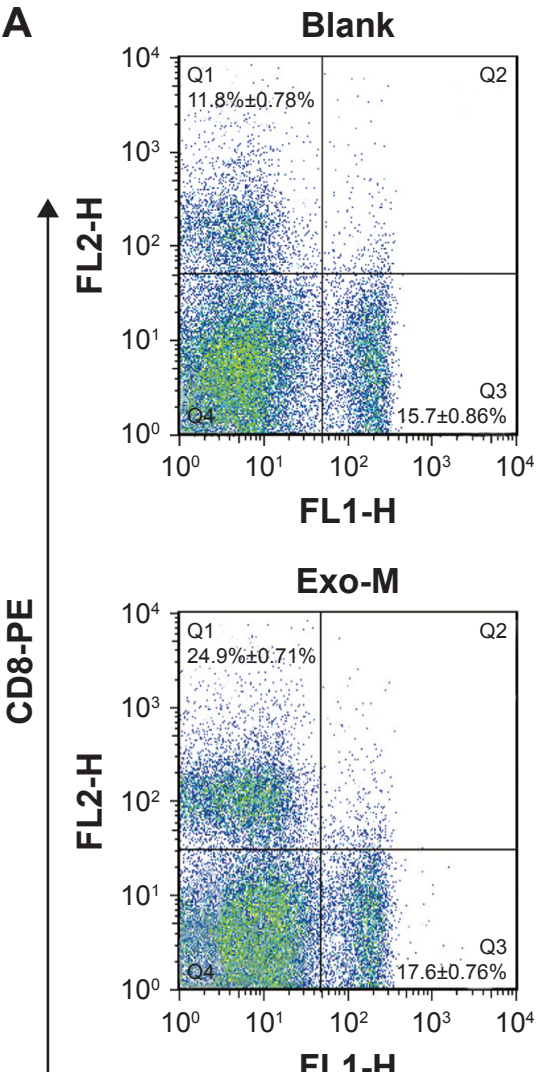

FL1-H
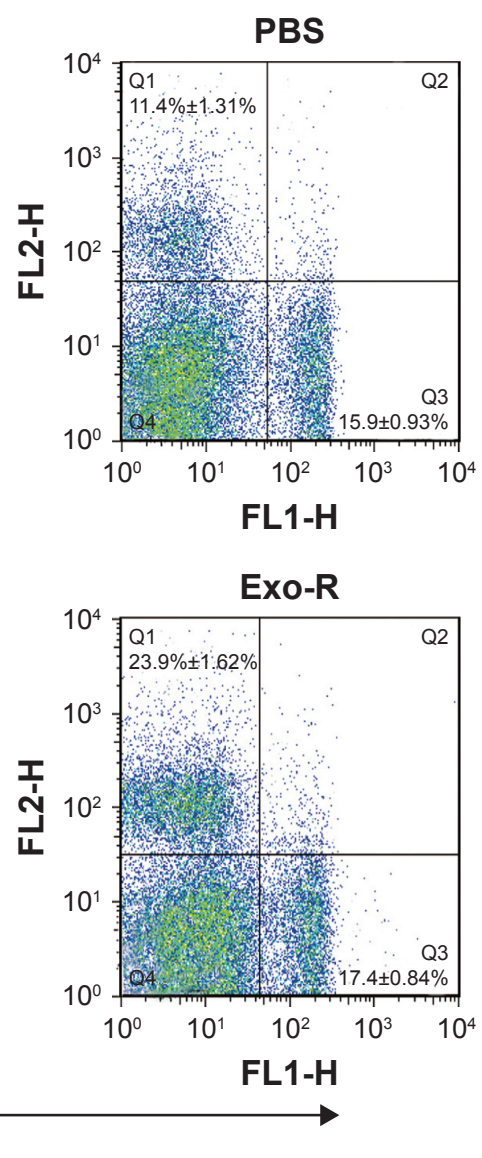

B

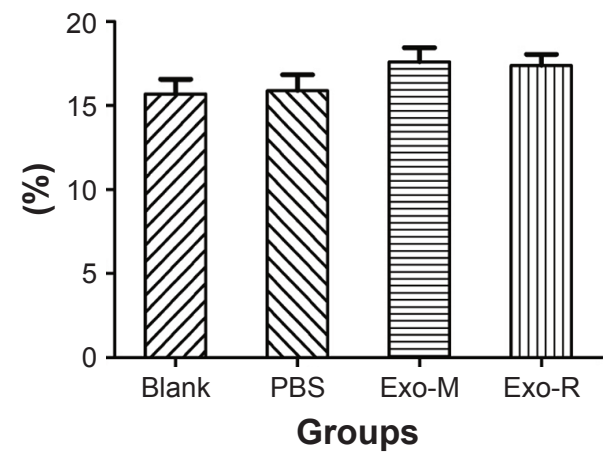

C

Percentages of CD8 ${ }^{+}$

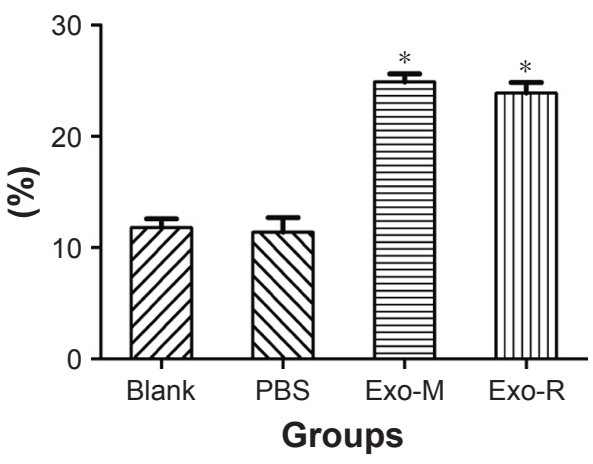

\section{CD4-FITC}

Figure 6 Flow cytometry analysis of T cell subsets.

Notes: The total mouse spleen cells were analyzed by using anti-mouse-CD3e-PE-cy5.5, anti-mouse-CD4-FITC and anti-mouse-CD8-PE for quality control. (A) The percentages of $\mathrm{CD}^{+}$(region Q3) and $\mathrm{CD}^{+}$(region QI) $\mathrm{T}$ cells in the spleen of immunized mice are shown and the results are representative of three independent experiments. (B) The percentages of $C D 4^{+}$and $(\mathbf{C})$ the percentages of $C D 8^{+}$. One of three individual experiments is shown. $n=4$ animals per group, ${ }^{*} p<0.05$ compared with PBS or Blank control.

Abbreviations: Exo-R, exosomes from RH strain; Exo-M, exosomes from ME 49 strain; FITC, fluorescein isothiocyanate; PE, phycoerythrin. 


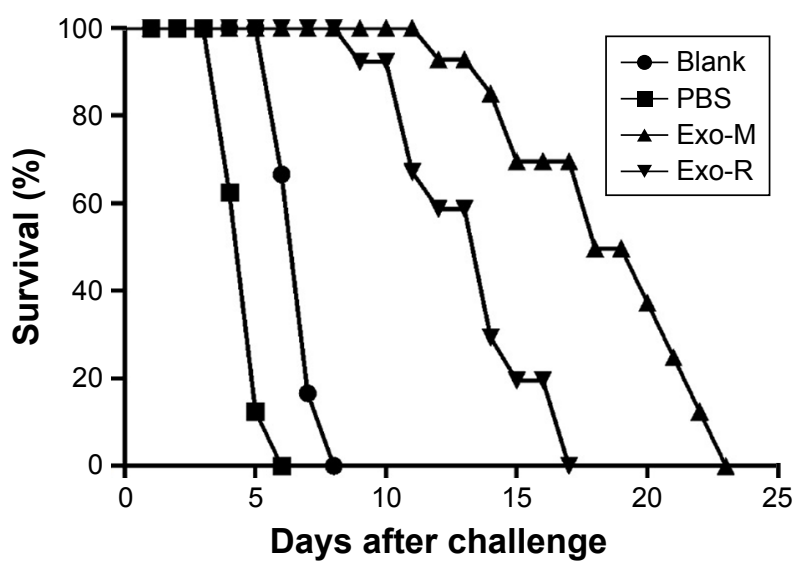

Figure 7 Survival curves of immunized mice after Toxoplasma gondii challenge infections.

Notes: $B A L B / c$ mice were challenged intraperitoneally with $I \times 10^{3}$ tachyzoites of T. gondii RH strain (PBS or Exo-R group) or ME 49 strain (Blank or Exo-M group) 2 weeks after the final immunization ( $n=10$ animals per group). Survival times were monitored daily and the comparison of differences between the experimental group and the control group was significant $(p<0.05)$. These data are representative of three experiments with similar results.

Abbreviations: Exo-R, exosomes from RH strain; Exo-M, exosomes from ME 49 strain; PBS, phosphate buffered saline.

terms of their biophysical and biological properties in their size, morphology, and protein components.

Increasing evidence has indicated that exosomes and their cargo can be transferred from one cell to another, resulting in the cell-to-cell communication via various functional biomolecules including proteins, bioactive lipids, and RNA, which can alter recipient cell functions. ${ }^{23-25}$ For instance, exosome-like vesicles from $T$. gondii-infected HFF cells could attach and deliver their contents to uninfected cells, thereby leading to altered functions in the uninfected cell. ${ }^{20}$ In addition, exosomes secreted by T. gondii-infected L6 cells could change the host cell proliferation and alter the host cell cycle. ${ }^{21}$ Similarly, our results demonstrated that T. gondii exosomes can be taken up by macrophages in vitro, suggesting the possible relevance of cell-to-cell communication based on exosomes. Importantly, exosomes were also shown to modulate in vitro cytokine productions. Previous study has revealed that exosomes from macrophages infected with different intracellular pathogens can stimulate macrophage activation in vitro, and that these exosomes contain PAMPs as an important mechanism of immune surveillance. ${ }^{19}$ Additionally, parasite-derived exosomes have been shown to promote immune cell activation, and induce cytokine production. ${ }^{11,13}$ Silverman et al have investigated the properties of exosomes from Leishmania donovani in terms of affecting innate and adaptive immune responses, resulting in high IFN- $\gamma$, high IL-10, and low TNF- $\alpha$ in human monocytes. ${ }^{11}$ Twu et al have reported that $T$. vaginalis exosomes specifically modulate the production of IL-8 rather than IL-6 production in human cervical epithelial cells. ${ }^{13}$ Herein, we demonstrated that $T$. gondii exosomes induced production of IL-12, IFN- $\gamma$ and TNF- $\alpha$ in macrophages, implying exosomes could stimulate macrophage activation via the production of proinflammatory cytokines. Moreover, as target cells, macrophages may be unsatisfactory since DCs are very important in activating $\mathrm{T}$ cells, more than macrophages. Future studies should consider those issues and address the molecular mechanisms by which $T$. gondii exosomes modulate host immune responses.

Currently, exosomes are under intensive exploration to realize their potential as therapeutic targets and diagnostic biomarkers. Previous studies have shown that exosomes can mediate immune responses including immune activation and immune suppression, and potentially trigger inflammation. ${ }^{11,26,27}$ Therefore, the potential use of exosomes as vaccines has aroused much interest. Mice treated with exosomes derived from DCs pulsed with $T$. gondii antigens were shown to elicit humoral and cellular immune response and protect the mice against subsequent parasite infection. ${ }^{17,18}$ Moreover, CBA/J mice immunized with SRDCs exosomes plus $T$. gondii antigens were induced protective immune responses against $T$. gondii infection in a congenital model. ${ }^{28}$ Consistent with these studies, our data showed that $T$. gondii exosomes could stimulate mice producing both humoral and cellular immune responses, and partial protective immunity against toxoplasmosis.

An ideal vaccine against toxoplasmosis should be sufficiently immunogenic to induce humoral and cellular immune responses, in particular, a potent Th1 type immune response. ${ }^{29}$ This Th1 immune response, defined by the production of IFN- $\gamma$ and IL-12, is characteristic of infection with many intracellular pathogens including T. gondii. IFN- $\gamma$ is a kind of critical cytokine in resisting $T$. gondii infection and promoting multiple intracellular mechanisms to kill the parasite and inhibit its replication. ${ }^{30}$ IFN- $\gamma$ also stimulates the generation of IgG2a and suppresses the synthesis of IgG1. In addition, IL-12 is a major cytokine for inducing Th1 type immune response and controlling the acute infection by $T$. gondii tachyzoites. ${ }^{31}$ In the present study, we demonstrated that mice immunized with $T$. gondii exosomes produced high levels of IFN- $\gamma$, IL-12, and IgG2a. However, there were no significant differences in the levels of IL-4 and IL-10 among all groups. These results indicated that $T$. gondii exosomes induced a dominant Th1 type immune response in mice, since IL-4, IL-10 and IgG1 were associated with Th2 type immune response. One possible mechanism of action 
might be attributable to the exosomes trafficking. It has been proved that exosomes can mediate antigen presentation and are implicated in the transfer of biological material from one cell to another. ${ }^{32}$ Therefore $T$. gondii exosomes could exchange the antigenic information between cells in the immune system and regulate the dynamic balance between Th1 and Th2 immune response.

Owing to the characteristics of the intracellular parasite, a strong $\mathrm{CD}^{+}$cytotoxic T lymphocyte (CTL) response plays an important role in the control of development and spreading of T. gondii infection. ${ }^{33,34}$ Our data showed that the percentage of $\mathrm{CD}^{+} \mathrm{T}$ cells was significantly increased in the immunized mice, suggesting that $T$. gondii exosomes might be presented by major histocompatibility complex (MHC) I molecules to $\mathrm{CD}^{+} \mathrm{T}$ cells. This was consistent with our previous observation in T cells' subsets activated by the recombinant DNA vaccine against $T$. gondii. ${ }^{16,35}$ Moreover, humoral immune response resulting in IgG antibodies seems to be necessary for preventing and controlling $T$. gondii infection. ${ }^{36}$ This is because specific antibodies can inhibit the attachment of the parasites to host cells, as well as promote macrophages to kill antibody-coated parasites. ${ }^{36}$

As far as the evaluation of protective potency of $T$. gondii exosomes was concerned, the inbred BALB/c mice were challenged intraperitoneally with $1 \times 10^{3}$ tachyzoites of $T$. gondii RH strain (Type I) and ME 49 strain (Type II). However, none of vaccinations provided complete protection against T. gondii infection, since all immunized mice died. Although the survival time of immunized mice between the two groups was different, there was no significant difference, which might be the virulence efficiency of $T$. gondii strains. It is known that RH strain of T. gondii is a highly virulent strain with $100 \%$ lethal dosage $(\mathrm{LD} 100)=1$ in mice while the ME49 strain is a low virulent strain $(50 \%$ lethal dosage $[$ LD50] $>$ or $=1,000)$ in mice. Besides, many parameters might hinder the evaluation of protection against $T$. gondii, including the dose of inoculums, the inoculation route, and the mouse strain. ${ }^{37}$ Future studies should consider the above factors as well as the tissue histology and parasite quantification, and also address the properties of exosomes and immunization strategy. Nonetheless, the $T$. gondii exosomes may be an effective alternative vaccine candidate against infectious disease.

\section{Conclusion}

In conclusion, we isolated the exosomes from $T$. gondii and identified the parasite-derived exosomes in their biophysical and biological properties. Functionally, we found that T. gondii exosomes could modulate macrophage activation in vitro. Furthermore, T. gondii exosomes triggered both humoral and cellular immune responses, and aroused partial protective immunity against acute parasite infection in the mice model. Therefore, this study suggested that exosomes may serve as a potential vaccine candidate or therapeutic agent against toxoplasmosis.

\section{Acknowledgments}

The authors wish to thank Dr Quan Liu and Dr Qijun Chen for kindly providing the ME49 strain of Toxoplasma gondii.

This study was financially supported by the National Natural Science Foundation of China (Grant No 81471974) and partly supported by Shandong Provincial Nature Foundation Science of China (Grant No ZR2011HZ003).

\section{Disclosure}

The authors report no conflicts of interest in this work.

\section{References}

1. Elmore SA, Jones JL, Conrad PA, et al. Toxoplasma gondii: epidemiology, feline clinical aspects, and prevention. Trends Parasitol. 2010; 26(4):190-196.

2. Montoya JG, Liesenfeld O. Toxoplasmosis. Lancet. 2004;363(9425): 1965-1976.

3. Weiss LM, Dubey JP. Toxoplasmosis: a history of clinical observations. Int J Parasitol. 2009;39(8):895-901.

4. Havelaar AH, Kemmeren JM, Kortbeek LM. Disease burden of congenital toxoplasmosis. Clin Infect Dis. 2007;44(11):1467-1474.

5. Tenter AM, Heckeroth AR, Weiss LM. Toxoplasma gondii: from animals to humans. Int J Parasitol. 2000;30(12):1217-1258.

6. Buxton D, Thomson K, Maley S, Wright S, Bos HJ. Vaccination of sheep with a live incomplete strain (S48) of Toxoplasma gondii and their immunity to challenge when pregnant. Vet Rec. 1991;129(5):89-93.

7. Schorey JS, Cheng Y, Singh PP, Smith PL. Exosomes and other extracellular vesicles in host-pathogen interactions. EMBO Rep. 2015; 16(1):24-43.

8. Coakley G, Maizels RM, Buck AH. Exosomes and other extracellular vesicles: the new communicators in parasite infections. Trends Parasitol. 2015;31(10):477-489.

9. Raposo G, Stoorvogel W. Extracellular vesicles: exosomes, microvesicles, and friends. J Cell Biol. 2013;200(4):373-383.

10. Théry C. Exosomes: secreted vesicles and intercellular communications. F1000 Biol Rep. 2011;3:15.

11. Silverman JM, Clos J, Horakova E, et al. Leishmania exosomes modulate innate and adaptive immune responses through effects on monocytes and dendritic cells. J Immunol. 2010;185(9):5011-5022.

12. Silverman JM, Reiner NE. Leishmania exosomes deliver preemptive strikes to create an environment permissive for early infection. Front Cell Infect Microbiol. 2012;1:26.

13. Twu $\mathrm{O}$, de Miquel N, Luatiq G, et al. Trichomonas vaginalis exosomes deliver cargo to host cells and mediate host:parasite interactions. PLoS Pathog. 2013;9(7):e1003482.

14. Wowk PF, Zardo ML, Miot HT, Goldenberg S, Carvalho PC, Mörking PA. Proteomic profiling of extracellular vesicles secreted from Toxoplasma gondii. Proteomics. 2017;17:15-16.

15. Cao A, Liu Y, Wang J, et al. Toxoplasma gondii: vaccination with a DNA vaccine encoding T- and B-cell epitopes of SAG1, GRA2, GRA7 and ROP16 elicits protection against acute toxoplasmosis in mice. Vaccine. 2015;33(48):6757-6762.

16. Muller L, Hong C, Stolz DB, Watkins SC, Whiteside TL. Isolation of biologically-active exosomes from human plasma. J Immunol Methods. 2014;411:55-65. 
17. Aline F, Bout D, Amigorena S, Roingeard P, Dimier-Poisson I. Toxoplasma gondii antigen-pulsed-dendritic cell-derived exosomes induce a protective immune response against $T$. gondii infection. Infect Immun. 2004;72(7):4127-4137.

18. Beauvillain C, Ruiz S, Guiton R, Bout D, Dimier-Poisson I. A vaccine based on exosomes secreted by a dendritic cell line confers protection against $T$. gondii infection in syngeneic and allogeneic mice. Microbes Infect. 2007;9(14-15):1614-1622.

19. Bhatnagar S, Shinagawa K, Castellino FJ, Schorey JS. Exosomes released from macrophages infected with intracellular pathogens stimulate a proinflammatory response in vitro and in vivo. Blood. 2007; 110(9):3234-3244.

20. Pope SM, Lässer C. Toxoplasma gondii infection of fibroblasts causes the production of exosome-like vesicles containing a unique array of mRNA and miRNA transcripts compared to serum starvation. J Extracell Vesicles. 2013;2(1):22484.

21. Kim MJ, Jung B, Cho J, et al. Exosomes secreted by Toxoplasma gondii-infected L6 cells: their effects on host cell proliferation and cell cycle changes. Korean J Parasitol. 2016;54(2):147-154.

22. Velge-Roussel F, Chardes T, Mevelec P, Brillard M, Hoebeke J, Bout D Epitopic analysis of the Toxoplasma gondii major surface antigen SAG1. Mol Biochem Parasitol. 1994;66(1):31-38.

23. Simons M, Raposo G. Exosomes - vesicular carriers for intercellular communication. Curr Opin Cell Biol. 2009;21(4):575-581.

24. Nazimek K, Bryniarski K, Santocki M, Ptak W. Exosomes as mediators of intercellular communication: clinical implications. Pol Arch Med Wewn. 2015;125(5):370-380.

25. Diaz G, Wolfe LM, Kruh-Garcia NA, Dobos KM. Changes in the membrane-associated proteins of exosomes released from human macrophages after mycobacterium tuberculosis infection. Sci Rep. 2016;6:37975.

26. Keller S, Sanderson MP, Stoeck A, Altevogt P. Exosomes: from biogenesis and secretion to biological function. Immunol Lett. 2006; 107(2):102-108.
27. Wei G, Jie Y, Haibo L, et al. Dendritic cells derived exosomes migration to spleen and induction of inflammation are regulated by CCR7. Sci Rep. 2017;7:42996.

28. Beauvillain C, Juste MO, Dion S, Pierre J, Dimier-Poisson I. Exosomes are an effective vaccine against congenital toxoplasmosis in mice. Vaccine. 2009;27(11):1750-1757.

29. Gazzinelli RT, Denkers EY, Sher A. Host resistance to Toxoplasma gondii: model for studying the selective induction of cell-mediated immunity by intracellular parasites. Infect Agents Dis. 1993;2(3):139-149.

30. Dupont CD, Christian DA, Hunter CA. Immune response and immunopathology during toxoplasmosis. Semin Immunopathol. 2012; 34(6):793-813.

31. Mashayekhi M, Sandau MM, Dunay IR, et al. CD8 $\alpha(+)$ dendritic cells are the critical source of interleukin-12 that controls acute infection by Toxoplasma gondii tachyzoites. Immunity. 2011;35(2):249-259.

32. Février B, Raposo G. Exosomes: endosomal-derived vesicles shipping extracellular messages. Curr Opin Cell Biol. 2004;16(4):415-421.

33. Gazzinelli RT, Eltoum I, Wynn TA, Sher A. Acute cerebral toxoplasmosis is induced by in vivo neutralization of TNF-alpha and correlates with the down-regulated expression of inducible nitric oxide synthase and other markers of macrophage activation. J Immunol. 1993 151(7):3672-3681.

34. Munoz M, Liesenfeld O, Heimesaat MM. Immunology of Toxoplasma gondii. Immunol Rev. 2011;240(1):269-285.

35. Min J, Qu D, Li C, et al. Enhancement of protective immune responses induced by Toxoplasma gondii dense granule antigen 7 (GRA7) against toxoplasmosis in mice using a prime-boost vaccination strategy. Vaccine. 2012;30(38):5631-5636

36. Sayles PC, Gibson GW, Johnson LL. B cells are essential for vaccination-induced resistance to virulent Toxoplasma gondii. Infect Immun. 2000;68(3):1026-1033.

37. Zhang NZ, Wang M, Xu Y, Petersen E, Zhu XQ. Recent advances in developing vaccines against Toxoplasma gondii: an update. Expert Rev Vaccines. 2015;14(12):1609-1621.
International Journal of Nanomedicine

\section{Publish your work in this journal}

The International Journal of Nanomedicine is an international, peerreviewed journal focusing on the application of nanotechnology in diagnostics, therapeutics, and drug delivery systems throughou the biomedical field. This journal is indexed on PubMed Central, MedLine, CAS, SciSearch $\AA$, Current Contents $₫ /$ Clinical Medicine,

\section{Dovepress}

Journal Citation Reports/Science Edition, EMBase, Scopus and the Elsevier Bibliographic databases. The manuscript management system is completely online and includes a very quick and fair peer-review system, which is all easy to use. Visit http://www.dovepress.com/ testimonials.php to read real quotes from published authors. 